\title{
Diagnostic and therapeutic strategies in cryptococcosis: impact on outcome
}

\author{
Timothée Boyer Chammard ${ }^{1}$, Elvis Temfack², Olivier Lortholary ${ }^{1,3}$, Alexandre Alanio ${ }^{1,4 /+}$ \\ ${ }^{1}$ Institut Pasteur, Centre National de Référence Mycoses Invasives et Antifongiques, CNRS CMR2000, Unité de Mycologie Moléculaire, Paris, France \\ ${ }^{2}$ Douala General Hospital, Internal Medicine Department, Douala, Cameroon \\ ${ }^{3}$ University Paris Descartes, Necker Pasteur Centre for Infectious Diseases and Tropical Medicine, Necker Hospital, Paris, France \\ ${ }^{4}$ University Paris Diderot, Sorbonne Paris City, Laboratory of Parasitology-Mycology, Saint-Louis Hospital, Paris, France
}

Cryptococcosis diagnosis has been recently improved by the use of rapid cryptococcal antigen testing with lateral flow assays, which have proved sensitive and specific. Using "test and treat" screening strategies for cryptococcal disease with these tests has been showed effective in reducing cryptococcal meningitis (CM) in HIV-infected patients. Recommended induction, consolidation, and maintenance therapeutic strategy for CM is widely unavailable and/or expensive in low and middle-income settings. New therapeutic strategies, mostly using reduced duration, have recently shown acceptable outcome or are currently tested. Diagnostic and therapeutic guidelines for cryptococcal disease in limited resources countries are undergoing a paradigmatic shift.

Key words: Cryptococcus neoformans - cryptococcal meningo-encephalitis - cryptococcal antigen - HIV infection - advanced AIDS disease screening

\section{DIAGNOSTIC STRATEGIES}

The diagnosis of cryptococcal meningitis $(\mathrm{CM})$ and more broadly, disseminated cryptococcosis, is easily made by direct examination of a pelleted biological fluid using India ink with the typical observation of rounded cells measuring about 3-10 $\mu \mathrm{m}$ in diameter surrounded a thick capsule represented as a clear halo. Sensitivity of this technique is around $86 \%$ (Kambugu et al. 2008, Boulware et al. 2014b) but can be lower in HIV-negative patients in association with a low fungal burden (Dromer et al. 2007). The definitive diagnosis relies on cultures of biological samples in Sabouraud dextrose agar incubated at $30^{\circ} \mathrm{C}$ (Arendrup et al. 2012). Culture might be negative in case of exposure to antifungal therapy or may need longer incubation periods up to three weeks. Another easy way to diagnose cryptococcosis is the detection of cryptococcal antigen (CrAg). $\mathrm{CrAg}$ is composed of polysaccharide of the cryptococcal capsule which is shed in biological fluids and can be detected as a marker of the presence of the fungus. Detection of $\mathrm{CrAg}$ relies usually on specific monoclonal antibodies using different methods among which are latex agglutination (LA), enzyme-linked immunosorbent assays (ELISA) and most recently a lateral flow assay (LFA). CrAg detection in serum is presumptive of active cryptococcosis, especially in HIV patients and detection in cerebrospinal fluid (CSF) is diagnostic of CM (Temstet et al. 1992).

However, cryptococcosis is a sub-acute infection. Consequently, CrAg can be detected in blood many weeks before the onset of symptoms (French et al. 2002).

doi: 10.1590/0074-02760180050

+ Corresponding author: alexandre.alanio@pasteur.fr

Received 29 January 2018

Accepted 19 March 2018
Serum CrAg positive patients are at 25\% higher risk of developing CM within the first year of ART (Letang et al. 2015, Meya et al. 2015). CrAg screening of patients before initiation of ART associated with preemptive fluconazole treatment once $\mathrm{CrAg}$ is positive was associated with low number of cases of CM (Longley et al. 2016). This combined strategy is associated with a significantly reduced mortality (Mfinanga et al. 2015).

As such, systematic pre-ART CrAg screening and preemptive fluconazole is recommended for those presenting with $<100$ CD4 cell counts (WHO 2017). Though promising as a strategy to decrease the incidence of cryptococcal disease, especially when associated with timely ART initiation, its implementation still lags in many countries.

CrAg detection and diagnosis of cryptococcal disease - Commercially available CrAg detection tests are highly sensitive and specific in serum and CSF in predicting the presence of meningitis in HIV positive patients presenting with evocative symptoms. CrAg detection in serum and CSF with symptoms suggestive of CM strongly correlates with culture- and/or India ink-confirmed CM. CSF $\mathrm{CrAg}$ is a rapid and reliable diagnostic test for confirmed HIV-associated CM (Temfack et al. 2018). In a recently published study, the new semi-quantitative BioSynex/ Bio-Rad CryptoPS ${ }^{\circledR}$ lateral flow assay at point of screening, clearly classified patients with or without CM (Temfack et al. 2018). Of note, serum and CSF CrAg should be preferentially considered at the time of diagnosis, as there is no clear correlation between changes in antigen titre and CSF cryptococcal culture (Brouwer et al. 2005). Persistence of detectable $\mathrm{CrAg}$ is frequent despite clinical improvement and complete recovery of the patient. The correlation between the evolution of $\mathrm{CrAg}$ levels during treatment and clinical improvement remains unclear and in case of early recurrence of symptoms, CSF CrAg should also be interpreted with caution. 
Pre-ART CrAg screening and pre-emptive therapy In HIV-infected patients with advanced AIDS disease, the prevalence of $\mathrm{CrAg}$ positivity ranged from $2-21 \%$, with a median of $6 \%$ (IQR: $5-7$ ) (Temfack et al., unpublished observations). CrAg-positive patients who were not offered fluconazole pre-emptive therapy are at higher risk of CM and death (Desmet et al. 1989, Jarvis et al. 2009). More so, about a third of CrAg-positive asymptomatic patients have evidence of CM (Wake et al. 2018). In CrAg positive patients who received preemptive fluconazole initiated at $800 \mathrm{mg} /$ day, there was a significant decrease in the relative risk of developing CM (from 21.4-6\%) and of incident mortality (Temfack et al., unpublished observations).

\section{THERAPEUTIC STRATEGIES}

The main challenge for cryptococcosis management is for disseminated disease and meningoencephalitis, knowing that the printciple of therapy is similar for both. For both clinical presentations, recommended first-line antifungal therapy is based on a three-steps strategy including induction, consolidation and maintenance phases, that was first used in a landmark Mycoses Study Group trial (van der Horst et al. 1997). The 2010 IDSA guidelines (Perfect et al. 2010) for management of disseminated disease placed amphotericin B and flucytosine as first choices for induction phase, with amphotericin B deoxycholate $(0.7-1.0 \mathrm{mg} / \mathrm{kg} / \mathrm{day})$ given intravenously in combination with oral flucytosine $100 \mathrm{mg} /$ $\mathrm{kg} /$ day (at least two weeks for HIV-infected patients and organ transplant recipients, and four to six weeks for all other patients). When available, the use of liposomal formulation of amphotericin B is preferred, as it has been showed to induce less toxicity. The preferred therapy for pulmonary-limited disease, or non-disseminated disease is based on fluconazole therapy alone.

The aim of CM therapy is to improve survival thanks to rapid yeast clearance from the CSF. Quantitative clearance from CSF, called "early fungicidal activity" (EFA), is measured as the rate of yeast clearance per millilitre of CSF per day based on CFU counting. In a combined cohort of 262 patients, a slow rate of cryptococcal clearance was shown to be independently associated with increased mortality at two weeks and at 10 weeks (Bicanic et al. 2009b). Though, in a recently published meta-analysis, combining 2854 HIV-infected or non HIV-infected patients in 27 randomised $\mathrm{CM}$ treatment trials, the authors concluded that all-cause mortality remains the essential primary outcome to be used to assess therapy efficacy in clinical trials (Montezuma-Rusca et al. 2016).

Thereafter, we summarise the main studies and clinical trials which have helped to improve cryptococcosis outcome and survival in the last decades.

\section{COMBINATION ANTIFUNGAL THERAPY}

Amphotericin B with flucytosine - The use of flucytosine combined with amphotericin B for CM therapy was already observed in a study in 1979, before the HIV pandemic, where authors concluded that the combination therapy allowed more rapid sterilisation of the cerebrospinal fluid $(\mathrm{p}<0.001)$ and less nephrotoxicity ( $p$
$<0.05)$ than did amphotericin B alone, although results were obtained at different dosages than those currently recommended (Bennett et al. 1979).

In 1997, the Mycoses Study Group randomised, double-blind, placebo-controlled, trial involving 408 HIV-infected patients evidenced that the use of a combination therapy with amphotericin B plus flucytosine for induction therapy was associated with an increased rate of CSF sterilisation and a better outcome than amphotericin B alone, in HIV-associated CM, although without improved survival (van der Horst et al. 1997).

Aiming to confirm these results, a randomised trial conducted in Thailand, also found that the efficacy of therapy (as measured by EFA) was shown to be significantly faster in the arm in which patients were treated with the combination of amphotericin B plus flucytosine than in any of the three other arms (amphotericin B alone, amphotericin B plus fluconazole, or a triple therapy with amphotericin $\mathrm{B}$, fluconazole and flucytosine), in 64 HIV-infected patients with CM (Brouwer et al. 2004).

This strategy was then also shown to be superior in the French prospective CryptoA/D study in HIV-positive or HIV-negative patients with cryptococcal infection with a mycological failure at week two of $26 \%$ in the amphotericin B and flucytosine group compared to a treatment failure of $56 \%$ with any other treatments $(\mathrm{p}<$ 0.001) (Dromer et al. 2007, Dromer et al. 2008).

Moreover, a three-arm, open-label, randomised trial including about 300 HIV-infected patients enrolled, demonstrated a survival benefit with this combination compared to amphotericin alone, with a reduction of about $40 \%$ in the relative risk of death at 10 weeks with addition of flucytosine (Day et al. 2013).

Finally, the recently completed "Advancing Cryptococcal Meningitis Treatment for Africa" (ACTA) trial, in which $721 \mathrm{HIV}$-infected patients with first episode of $\mathrm{CM}$ were randomised over nine sites in four different countries in Sub-Saharan Africa, definitively evidenced that the combination of amphotericin B and flucytosine was significantly superior to Amphotericin B and fluconazole, leading to a substantial mortality reduction [hazard ratio for death at 10 weeks with flucytosine vs. fluconazole, $0.62 ; 95 \%$ confidence interval (CI), 0.45 to 0.84; $\mathrm{p}=0.002$ ] (Molloy et al. 2018).

Amphotericin B with fluconazole - When flucytosine is unavailable, the combination of amphotericin $B$ with fluconazole is still recommended (Perfect et al. 2010). In an open-label, three-arm, phase II trial including 143 HIVinfected patients, combination of amphotericin B with fluconazole $800 \mathrm{mg} /$ day was found to have significantly better long-term outcomes than amphotericin B and fluconazole $400 \mathrm{mg} /$ day or amphotericin alone (Pappas et al. 2009). However, this have not been observed in the study conducted by Day at al. (2013), with no statistically survival benefit found for patients receiving amphotericin with fluconazole $800 \mathrm{mg} /$ day compared to those with amphotericin B alone. More so, in the ACTA trial, the treatment arm combining Amphotericin B and fluconazole had the worse outcome, clearly demonstrating that the combination of Amphotericin $\mathrm{B}$ and fluconazole is a not recommended for induction treatment (Molloy et al. 2018). 
Amphotericin B with voriconazole - The use of voriconazole at the dosage of $300 \mathrm{mg}$ twice daily, instead of highdose fluconazole ( $800 \mathrm{mg}$ or $1200 \mathrm{mg} /$ day) in induction therapy associated with amphotericin B was tested in a randomised 4-arms trial and found to have similar EFA, and could therefore be a theoretical option for therapy, despite its higher cost and potential interactions with other drugs including rifampicin (Loyse et al. 2012). As voriconazole is not available in most of the countries endemic for cryptococcosis, this combination would be marginally used.

Short courses therapy - The amphotericin B and flucytosine combination regimen remains unavailable in most parts of the world harbouring the highest burden of disease (Africa and Asia), furthermore several days of amphotericin B deoxycholate is well known to cause important adverse events, such as anaemia, renal impairment, hypokalaemia, hypomagnesemia, or phlebitis, and its administration requires inpatient hospitalisation for intravenous administration and electrolytes monitoring (Bicanic et al. 2015).

Researcher in lower middle-income settings are now working to improve induction therapy phase with combination therapy, using oral combination or reducing duration of intravenous drugs, to reduce incidence of adverse events, and try to improve survival.

In a study in Uganda, based on $30 \mathrm{HIV-infected} \mathrm{pa-}$ tients, a short 5-day course of amphotericin with highdose fluconazole $1200 \mathrm{mg} /$ day was found to have a better EFA than findings with fluconazole alone in previous studies, suggesting that shorter courses of amphotericin may be used (Muzoora et al. 2012).

The multi-centre ACTA trial, testing short courses amphotericin B deoxycholate based therapies with either flucytosine or fluconazole, also showed a better survival with a 7-day course of amphotericin B and flucytosine compared with 14-day course (HR $0.56(0.35-0.91)$ ) (Molloy et al. 2018). This prompted WHO experts to now recommend a 7-days amphotericin B IV therapy with flucytosine as first line induction therapy (followed by seven days of high- dose fluconazole) in low and middle-income countries (WHO 2018).

Fluconazole and flucytosine - Before the highly active antiretroviral therapy era, an open-label, single-arm, prospective trial studied the fluconazole ( $400 \mathrm{mg}$ daily) and flucytosine $(150 \mathrm{mg} / \mathrm{kg}$ daily) combination therapy for a 10 -week antifungal therapy in 32 patients with AIDS, and found a clinical survival at 10 weeks which appeared to be better than previously reported with amphotericin $\mathrm{B}$ alone or fluconazole alone (Larsen et al. 1994).

More recently, a randomised clinical trial compared combination therapy with high dose fluconazole (1200 $\mathrm{mg}$ daily) and flucytosine (100 $\mathrm{mg} / \mathrm{kg}$ daily) to high dose fluconazole alone for the 2-week induction therapy, in 41 HIV-infected patients with CM in Malawi, and found better EFA and survival for the combination therapy arm (Nussbaum et al. 2010).

Finally, the ACTA trial including 721 randomised HIV-infected patients also showed that a combination oral therapy with high dose fluconazole $(1200 \mathrm{mg} /$ day $)$ and flucytosine $(100 \mathrm{mg} / \mathrm{kg} / \mathrm{day})$ for the 2 -week induction therapy was non-inferior to the standard 2 -week courses of amphotericin B based therapy with flucytosine or fluconazole (Molloy et al. 2018). That made authors conclude that this regimen is an acceptable and effective option for CM therapy in Africa if amphotericin $\mathrm{B}$ is not available or not recommended.

\section{FLUCONAZOLE MONOTHERAPY}

Both 2010 IDSA and 2011 WHO guidelines recommend high-dose fluconazole monotherapy $(1200 \mathrm{mg} /$ day) for 10-12 weeks if amphotericin and flucytosine are not available (Perfect et al. 2010, WHO 2011).

In a cohort study carried out in Malawi using high dose fluconazole (1200 mg) as induction therapy (Gaskell et al. 2014), mortality was unacceptably high, as it was in a study using $800 \mathrm{mg}$ (Rothe et al. 2013), showing that fluconazole monotherapy is not suitable as induction therapy.

A recently published CrAg screening study in Ethiopia in HIV-infected patients, highlighted that the use of high dose fluconazole monotherapy for induction phase is inadequate, leading to a $68 \%$ mortality rate at three months, in patients with CM (Beyene et al. 2017).

\section{USE OF LIPOSOMAL AMPHOTERICIN B}

At the beginning of highly active antiretroviral therapy era, in 1997, a randomised trial comparing liposomal amphotericin B (L-AmB) and conventional deoxycholate amphotericin B in $28 \mathrm{HIV}$-infected patients with $\mathrm{CM}$, observed that a 3 -week course of $4 \mathrm{mg} / \mathrm{kg} \mathrm{L}-\mathrm{AmB}$ had a significantly earlier CSF culture conversion than a 3-week course standard amphotericin B, with similar clinical outcome and that L-AmB had significantly less renal adverse events (Leenders et al. 1997).

In a larger multi-centre trial, in which 267 patients were randomised, liposomal amphotericin B - therapy was similar in term of efficacy and overall mortality at 10 weeks, compared to conventional amphotericin B deoxycholate for HIV-associated CM. L-AmB at a dosage of $3 \mathrm{mg} / \mathrm{kg} /$ day was associated with significantly fewer adverse effects than at a dosage of $6 \mathrm{mg} / \mathrm{kg} / \mathrm{day}$, and compared to conventional amphotericin B (Hamill et al. 2010).

More recently, 80 participants were enrolled in the AMBITION preliminary phase II trial comparing different short courses of L-AmB with high dose fluconazole. The single dose arm was non-inferior to the standard 14days arm in term of early fungicidal activity, compared to two-doses or three-doses arms (Jarvis et al. 2017).

Based on these results, the AMBITION phase III (ISRCTN 72509687) ongoing trial is currently taking place to evaluate whether a single high dose $(10 \mathrm{mg} / \mathrm{kg})$ of L$\mathrm{AmB}$ given with high dose fluconazole and flucytosine is as effective as the newly recommended (based on ACTA trial) one-week daily-dosed amphotericin B deoxycholate based induction therapy with flucytosine as an induction therapy (Molefi et al. 2015). This trial will be the largest therapeutic trial on $\mathrm{CM}$ with 850 patients to be enrolled over a 3-year period in five different African countries.

\section{ADJUVANT THERAPIES}

Steroids - Adjuvant glucocorticoid therapy is successfully used in patients with non-fungal meningitis such as bacterial or tuberculous meningitis, and reduces mortality 
in some sub-groups. In CM IDSA guidelines (Perfect et al. 2010), experts recommend to consider the use of dexamethasone at high doses for patients having immune reconstitution inflammatory syndrome with severe central nervous system signs and symptoms, using a two-six-week course.

The CRYPTODEX double-blind, randomised, placebo-controlled trial, conducted in 6 countries over Africa and Asia, aimed to demonstrate the efficacy of adjunctive dexamethasone but was stopped after the enrolment of 451 patients (on an overall of 880 planned) for safety reasons (Beardsley et al. 2016). All patients received the locally-available and standard-of-care combination antifungal therapy with amphotericin B and fluconazole, and were randomised to receive either dexamethasone (intravenous for the first two weeks, and then orally, tapered doses until the 6th week) or placebo for six weeks. The primary outcome was survival until 10 weeks after randomisation. There was no difference between groups - 47\% in the dexamethasone group and $41 \%$ in the placebo group died. EFA was measured and during the first two weeks of treatment, dexamethasone was significantly associated with slower rates of decline in the EFA in CSF than was placebo. Even if dexamethasone was significantly associated with a better reduction in CSF opening pressure during the first two weeks than was placebo, it was also associated with more adverse events and disability.

Interferon-gamma - In a prospective trial, with 90 HIV-infected patients randomised in three different arms [standard therapy with amphotericin B plus flucytosine, standard therapy with two doses of interferon-gamma (INF- $\gamma$ ), and standard therapy with six doses of INF- $\gamma$ ], an increased rate of clearance with two doses of adjunctive INF- $\gamma$ than with standard therapy was shown, but there was no significant difference in mortality between groups (Jarvis et al. 2012).

Sertraline - Previous studies have suggested that sertraline, a selective serotonin reuptake inhibitor (SSRI), frequently used as an anti-depressant, has a real in vitro and in vivo fungicidal activity against Cryptococcus neoformans, as its action has a synergistic effect with fluconazole in reducing the fungal burden in brain, kidney, and spleen (Zhai et al. 2012). The first clinical open-label and dose-finding study was conducted in Uganda, recruiting 172 patients with HIV-associated CM, to assess the efficacy of adjunctive sertraline, with standard amphotericin B and high-dose fluconazole antifungal therapies (Rhein et al. 2016). Authors concluded that patients had faster cryptococcal CSF clearance and a lower incidence of immune reconstitution inflammatory syndrome and relapse than that reported in the past.

Following these results, the "Adjunctive Sertraline for the Treatment of HIV-associated Cryptococcal Meningitis (ASTRO-CM)" phase III randomised placebo-controlled clinical trial had been evaluating if sertraline dosed initially at $400 \mathrm{mg}$ /day for two weeks, followed by $200 \mathrm{mg}$ /day for an additional 10 weeks prior to tapering, could have an 18-week survival benefit, compared with placebo when receiving standard available induction therapy of amphotericin B deoxycholate and fluconazole $800 \mathrm{mg} /$ day. The trial was stopped after enrolling 460 of the 550 patients planned for futility, showing a 18 -week mortality of $52 \%$ in sertraline group and $46 \%$ in placebo group $(\mathrm{p}=0.15)$ (Rhein et al. 2018).

Tamoxifen - Tamoxifen, the oestrogen receptor antagonist drug usually used for breast cancer, has been shown to be fungicidal and synergistic with fluconazole and amphotericin B in vitro and in vivo in a mouse model of disseminated cryptococcosis (Butts et al. 2014). At acceptably concentrations for humans, tamoxifen combined with fluconazole decreased brain fungal burden, and has demonstrated to inhibit the growth of $C$. neoformans within macrophages, which are not accessible by classical antifungal drugs. It has also the benefit to have a good oral bioavailability.

Therefore, an open-label, phase II, randomised trial ("A randomised trial of tamoxifen combined with amphotericin B and fluconazole for cryptococcal meningitis", NCT03112031) will enrol 50 patients in Vietnam, comparing EFA according to the strategy between (amphotericin B plus fluconazole \pm tamoxifen during the first two weeks).

\section{ELEVATED OPENING CEREBROSPINAL FLUID PRESSURE}

Elevated intracranial pressure (CSF pressure $\geq 25 \mathrm{~cm}$ $\mathrm{H} 2 \mathrm{O}$ ) is a usual complication of CM, secondary to a failure of CSF resorption because of obstruction by cryptococcal polysaccharide capsule and yeasts.

In several large randomised trials (van der Horst et al. 1997, Brouwer et al. 2004, Bicanic et al. 2007) of amphotericin B-based therapy for CM, the effect of baseline CSF opening pressure was evaluated (Graybill et al. 2000, Bicanic et al. 2009a) and led to recommend to relieve by large-volume CSF drainage, performing daily therapeutic LPs if the CSF pressure is $\geq 25 \mathrm{~cm}$ of CSF with symptoms of increased intracranial pressure during induction therapy.

Another randomised trial comparing acetazolamide or placebo, with amphotericin B alone was prematurely stopped after recruitment of 24 patients in Thailand with $\mathrm{CM}$, presenting with headache and an elevated opening cerebrospinal fluid pressure $\geq 20 \mathrm{~cm} \mathrm{H} 20$, after having observed that patients who received acetazolamide developed significantly lower venous bicarbonate levels and higher chloride levels and had more frequent serious adverse events than patients who received placebo (Newton et al. 2002).

In the COAT trial, the effect of therapeutic lumbar puncture (LP) was evaluated in HIV-infected patients treated for $\mathrm{CM}$, and investigators found that therapeutic LPs were associated with a $69 \%$ relative improvement in survival, regardless of initial intracranial pressure (Boulware et al. 2014a, Rolfes et al. 2014).

\section{ANTIRETROVIRAL THERAPY FOR HIV-INFECTED PATIENTS}

Antiretroviral therapy (ART) is the corner stone of treatment in HIV-infected patients with advanced AIDS disease. In patients with $\mathrm{CM}$, it is now recommended delaying ART for four-six weeks after starting antifungal therapy (WHO 2017). This deferring strategy has indeed shown to significantly improve survival, compared to early ART initiation strategy (Boulware et al. 2014a). 


\section{NON-HIV-INFECTED PATIENTS}

In solid organ transplant recipients, cryptococcal disease has different presentation, with less disseminated disease, and more lung-limited presentation (Singh et al. 2008). This could be caused by the use of calcineurin inhibitor immunosuppressive agents - such as cyclosporine or tacrolimus. These agents are synergistic with antifungals, and their use had shown better outcomes (Kontoyiannis et al. 2008). The optimal way to manage immunosuppressive regimen is to do sequential or stepwise reduction of immunosuppressive agents, and to consider decreasing corticosteroid therapy first (Perfect et al. 2010). For disseminated presentations, lipid formulations of amphotericin B are preferred to standard amphotericin B deoxycholate based therapy, given the potential risk of renal deterioration and drug interactions in these patients.

\section{IN CONCLUSION}

Cryptococcal disease is a death-related disease in immunosuppressed patients, especially in advanced AIDS disease patients. Over the past 15 years, many studies and clinical trials have led to improve prevention, diagnosis, therapy and outcome of patients with cryptococcal meningitis. Efforts are now needed to implement those strategies in middle and low-income settings using affordable and efficient diagnostic strategies and drug regimen. These new strategies are being evaluated and will be implemented progressively to improve patient management.

\section{AUTHORS' CONTRIBUTION}

TBC, ET and AA wrote the paper; OL and ET - major criticism of the manuscript.

\section{REFERENCES}

Arendrup MC, Bille J, Dannaoui E, Ruhnke M, Heussel C-P, Kibbler C. ECIL-3 classical diagnostic procedures for the diagnosis of invasive fungal diseases in patients with leukaemia. Bone Marrow Transplant. 2012; 47(8): 1030-45.

Beardsley J, Wolbers M, Kibengo FM, Ggayi A-BM, Kamali A, Cuc NTK, et al. Adjunctive dexamethasone in HIV-associated cryptococcal meningitis. N Engl J Med. 2016; 374(6): 542-54.

Bennett JE, Dismukes WE, Duma RJ, Medoff G, Sande MA, Gallis $\mathrm{H}$, et al. A comparison of amphotericin B alone and combined with flucytosine in the treatment of cryptococcal meningitis. N Engl J Med. 1979; 19301(3): 126-31.

Beyene T, Zewde AG, Balcha A, Hirpo B, Yitbarik T, Gebissa T, et al. Inadequacy of high-dose fluconazole monotherapy among cerebrospinal fluid cryptococcal antigen ( $\mathrm{CrAg}$ )-positive human immunodeficiency virus-infected persons in an Ethiopian $\mathrm{CrAg}$ screening program. Clin Infect Dis. 2017; 17: 1-4.

Bicanic T, Bottomley C, Loyse A, Brouwer AE, Muzoora C, Taseera $\mathrm{K}$, et al. Toxicity of amphotericin B deoxycholate-based induction therapy in patients with HIV-associated cryptococcal meningitis. Antimicrob Agents Chemother. 2015; 59(12): 7224-31.

Bicanic T, Brouwer AE, Meintjes G, Rebe K, Limmathurotsakul D, Chierakul W, et al. Relationship of cerebrospinal fluid pressure, fungal burden and outcome in patients with cryptococcal meningitis undergoing serial lumbar punctures. AIDS. 2009a; 2723(6): 701-6.

Bicanic T, Meintjes G, Wood R, Hayes M, Rebe K, Bekker L-G, et al. Fungal burden, early fungicidal activity, and outcome in cryptococcal meningitis in antiretroviral-naive or antiretroviral-experienced patients treated with amphotericin B or fluconazole. Clin
Infect Dis. 2007; 145(1): 76-80.

Bicanic T, Muzoora C, Brouwer AE, Meintjes G, Longley N, Taseera $\mathrm{K}$, et al. Independent association between rate of clearance of infection and clinical outcome of HIV-associated cryptococcal meningitis: analysis of a combined cohort of 262 patients. Clin Infect Dis. 2009b; 149(5): 702-9.

Boulware DR, Meya DB, Muzoora C, Rolfes MA, Hullsiek KH, Musubire A, et al. Timing of antiretroviral therapy after diagnosis of cryptococcal meningitis. N Engl J Med. 2014a; 26370(26): 2487-98.

Boulware DR, Rolfes MA, Rajasingham R, Hohenberg von M, Qin $\mathrm{Z}$, Taseera $\mathrm{K}$, et al. Multisite validation of cryptococcal antigen lateral flow assay and quantification by laser thermal contrast. Emerg Infect Dis. 2014b; 20(1): 45-53.

Brouwer AE, Rajanuwong A, Chierakul W, Griffin GE, Larsen RA, White NJ, et al. Combination antifungal therapies for HIV-associated cryptococcal meningitis: a randomised trial. Lancet. 2004; 29363(9423): 1764-7.

Brouwer AE, Teparrukkul P, Pinpraphaporn S, Larsen RA, Chierakul $\mathrm{W}$, Peacock S, et al. Baseline correlation and comparative kinetics of cerebrospinal fluid colony-forming unit counts and antigen titers in cryptococcal meningitis. J Infect Dis. 2005; 15192(4): 681-4.

Butts A, Koselny K, Chabrier-Roselló Y, Semighini CP, Brown JCS, Wang $X$, et al. Estrogen receptor antagonists are anti-cryptococcal agents that directly bind $\mathrm{EF}$ hand proteins and synergize with fluconazole in vivo. MBio. 2014; 115(1): e00765-13.

Day JN, Chau TTH, Wolbers M, Mai PP, Dung NT, Mai NH, et al. Combination antifungal therapy for cryptococcal meningitis. $\mathrm{N}$ Engl J Med. 2013; 4368(14): 1291-302.

Desmet P, Kayembe KD, de Vroey C. The value of cryptococcal serum antigen screening among HIV-positive/AIDS patients in Kinshasa, Zaire. AIDS. 1989; 3(2): 77-8.

Dromer F, Bernede-Bauduin C, Guillemot D, Lortholary O, French Cryptococcosis Study Group. Major role for amphotericin B-flucytosine combination in severe cryptococcosis. PLoS One. 2008; 3(8): e2870.

Dromer F, Mathoulin-Pélissier S, Launay O, Lortholary O, French Cryptococcosis Study Group. Determinants of disease presentation and outcome during cryptococcosis: the CryptoA/D study. PLoS Med. 2007; 4(2): e21.

French N, Gray K, Watera C, Nakiyingi J, Lugada E, Moore M, et al. Cryptococcal infection in a cohort of HIV-1-infected Ugandan adults. AIDS. 2002; 316(7): 1031-8.

Gaskell KM, Rothe C, Gnanadurai R, Goodson P, Jassi C, Heyderman $\mathrm{RS}$, et al. A prospective study of mortality from cryptococcal meningitis following treatment induction with $1200 \mathrm{mg}$ oral fluconazole in Blantyre, Malawi. PLoS ONE. 2014; 9(11): e110285.

Graybill JR, Sobel J, Saag M, van der Horst C, Powderly W, Cloud $\mathrm{G}$, et al. Diagnosis and management of increased intracranial pressure in patients with AIDS and cryptococcal meningitis. The NIAID Mycoses Study Group and AIDS Cooperative Treatment Groups. Clin Infect Dis. 2000; 30(1): 47-54.

Hamill RJ, Sobel JD, El-Sadr W, Johnson PC, Graybill JR, Javaly K, et al. Comparison of 2 doses of liposomal amphotericin B and conventional amphotericin B deoxycholate for treatment of AIDS-associated acute cryptococcal meningitis: a randomized, double-blind clinical trial of efficacy and safety. Clin Infect Dis. 2010; 1551(2): 225-32.

Jarvis JN, Lawn SD, Vogt M, Bangani N, Wood R, Harrison TS. Screening for cryptococcal antigenemia in patients accessing an antiretroviral treatment program in South Africa. Clin Infect Dis. 2009; 48(7): 856-62.

Jarvis JN, Leeme TB, Chofle AA, Bidwell G, Molefi M, Tsholo K, et al. Ambition-cm: high-dose liposomal amphotericin for HIVrelated cryptococcal meningitis. In: Conference on retroviruses and opportunistic infections. Seattle, Feb 13-16 2017. 
Jarvis JN, Meintjes G, Rebe K, Williams GN, Bicanic T, Williams A, et al. Adjunctive interferon- $\gamma$ immunotherapy for the treatment of HIV-associated cryptococcal meningitis. AIDS. 2012; 26(9): 1105-13.

Kambugu A, Meya DB, Rhein J, O'Brien M, Janoff EN, Ronald AR, et al. Outcomes of cryptococcal meningitis in Uganda before and after the availability of highly active antiretroviral therapy. Clin Infect Dis. 2008; 46(11): 1694-701.

Kontoyiannis DP, Lewis RE, Alexander BD, Lortholary O, Dromer F, Gupta KL, et al. Calcineurin inhibitor agents interact synergistically with antifungal agents in vitro against Cryptococcus neoformans isolates: correlation with outcome in solid organ transplant recipients with cryptococcosis. Antimicrob Agents Chemother. 2008; 2352(2): 735-8.

Larsen RA, Bozzette SA, Jones BE, Haghighat D, Leal MA, Forthal $\mathrm{D}$, et al. Fluconazole combined with flucytosine for treatment of cryptococcal meningitis in patients with AIDS. Clin Infect Dis. 1994; 19(4): 741-5.

Leenders AC, Reiss P, Portegies P, Clezy K, Hop WC, Hoy J, et al. Liposomal amphotericin $\mathrm{B}$ (AmBisome) compared with amphotericin $B$ both followed by oral fluconazole in the treatment of AIDS-associated cryptococcal meningitis. AIDS. 1997; 11(12): 1463-71.

Letang E, Müller MC, Ntamatungiro AJ, Kimera N, Faini D, Furrer H, et al. Cryptococcal antigenemia in immunocompromised human immunodeficiency virus patients in rural Tanzania: a preventable cause of early mortality. Open Forum Infect Dis. 2015; 22(2): ofv046.

Longley N, Jarvis JN, Meintjes G, Boulle A, Cross A, Kelly N, et al. Cryptococcal antigen screening in patients initiating ART in South Africa: a prospective cohort study. Clin Infect Dis. 2016; 162(5): 581-7.

Loyse A, Wilson D, Meintjes G, Jarvis JN, Bicanic T, Bishop L, et al. Comparison of the early fungicidal activity of high-dose fluconazole, voriconazole, and flucytosine as second-line drugs given in combination with amphotericin B for the treatment of HIV-associated cryptococcal meningitis. Clin Infect Dis. 2012; 154(1): 121-8.

Meya D, Rajasingham R, Nalintya E, Tenforde M, Jarvis JN. Preventing cryptococcosis - Shifting the paradigm in the era of highly active antiretroviral therapy. Curr Trop Med Rep. 2015; 212(2): 81-9.

Mfinanga S, Chanda D, Kivuyo SL, Guinness L, Bottomley C, Simms V, et al. Cryptococcal meningitis screening and community-based early adherence support in people with advanced HIV infection starting antiretroviral therapy in Tanzania and Zambia: an open-label, randomised controlled trial. Lancet. 2015; 30385(9983): 2173-82.

Molefi M, Chofle AA, Molloy SF, Kalluvya S, Changalucha JM, Cainelli F, et al. AMBITION-cm: intermittent high dose AmBisome on a high dose fluconazole backbone for cryptococcal meningitis induction therapy in sub-Saharan Africa: study protocol for a randomized controlled trial. Trials. 2015; 1716(1): 276.

Molloy SF, Kanyama C, Heyderman RS, Loyse A, Kouanfack C, Chanda $\mathrm{D}$, et al. Antifungal combinations for treatment of cryptococcal meningitis in Africa. N Engl J Med. 2018; 378(11): 1004-17.

Montezuma-Rusca JM, Powers JH, Follmann D, Wang J, Sullivan B, Williamson PR. Early fungicidal activity as a candidate surrogate endpoint for all-cause mortality in cryptococcal meningitis: a systematic review of the evidence. PLoS One. 2016; 11(8): e0159727-15.

Muzoora CK, Kabanda T, Ortu G, Ssentamu J, Hearn P, Mwesigye J, et al. Short course amphotericin B with high dose fluconazole for HIV-associated cryptococcal meningitis. J Infect. 2012; 64(1): 76-81.

Newton PN, Thai LH, Tip NQ, Short JM, Chierakul W, Rajanuwong A, et al. A randomized, double-blind, placebo-controlled trial of acetazolamide for the treatment of elevated intracranial pressure in cryptococcal meningitis. Clin Infect Dis. 2002; 35(6): 769-72.

Nussbaum JC, Jackson A, Namarika D, Phulusa J, Kenala J, Kanyemba $\mathrm{C}$, et al. Combination flucytosine and high-dose fluconazole compared with fluconazole monotherapy for the treatment of cryptococcal meningitis: a randomized trial in malawi. Clin Infect Dis. 2010; 50(3): 338-44.

Pappas PG, Chetchotisakd P, Larsen RA, Manosuthi W, Morris MI, Anekthananon T, et al. A phase II randomized trial of amphotericin B alone or combined with fluconazole in the treatment of HIV-associated cryptococcal meningitis. Clin Infect Dis. 2009; 48(12): 1775-83.

Perfect JR, Dismukes WE, Dromer F, Goldman DL, Graybill JR, Hamill RJ, et al. Clinical Practice guidelines for the management of cryptococcal disease: 2010 update by the Infectious Diseases Society of America. Clin Infect Dis. 2010; 50(3): 291-322.

Rhein J, Hullsiek KH, Tugume L, Nuwagira E, Mpoza E, Kiggundu $\mathrm{R}$, et al. Adjunctive sertraline in HIV-associated cryptococcal meningitis. In: Conference on retroviruses and opportunistic infections. Boston, Mar 4-7 2018.

Rhein J, Morawski BM, Hullsiek KH, Nabeta HW, Kiggundu R, Tugume L, et al. Efficacy of adjunctive sertraline for the treatment of HIV-associated cryptococcal meningitis: an open-label dose-ranging study. Lancet Infect Dis. 2016; 16(7): 809-18.

Rolfes MA, Hullsiek KH, Rhein J, Nabeta HW, Taseera K, Schutz C, et al. The Effect of therapeutic lumbar punctures on acute mortality from cryptococcal meningitis. Clin Infect Dis. 2014; 59(11): 1607-14.

Rothe C, Sloan DJ, Goodson P, Chikafa J, Mukaka M, Denis B, et al. A prospective longitudinal study of the clinical outcomes from cryptococcal meningitis following treatment induction with $800 \mathrm{mg}$ oral fluconazole in Blantyre, Malawi. PLoS One. 2013; 8(6): e67311.

Singh N, Dromer F, Perfect JR, Lortholary O. Cryptococcosis in solid organ transplant recipients: current state of the science. Clin Infect Dis. 2008; 47(10): 1321-7.

Temfack E, Kouanfack C, Mossiang L, Loyse A, Fonkoua MC, Molloy SF, et al. Cryptococcal antigen screening in asymptomatic HIV-infected antiretroviral Naive patients in Cameroon and evaluation of the new semi-quantitative Biosynex CryptoPS Test. Front Microbiol. 2018; 13: 9.

Temstet A, Roux P, Poirot JL, Ronin O, Dromer F. Evaluation of a monoclonal antibody-based latex agglutination test for diagnosis of cryptococcosis: comparison with two tests using polyclonal antibodies. J Clin Microbiol. 1992; 30(10): 2544-50.

van der Horst CM, Saag MS, Cloud GA, Hamill RJ, Graybill JR, Sobel JD, et al. Treatment of cryptococcal meningitis associated with the acquired immunodeficiency syndrome. National Institute of Allergy and Infectious Diseases Mycoses Study Group and AIDS Clinical Trials Group. N Engl J Med. 1997; 337(1): 15-21.

Wake RM, Britz E, Sriruttan C, Rukasha I, Omar T, Spencer DC, et al. High cryptococcal antigen titers in blood are predictive of subclinical cryptococcal meningitis among HIV-infected patients. Clin Infect Dis. 2018; 66(5): 686-92.

WHO - World Health Organization. Guidelines for managing advanced HIV disease and rapid initiation of antiretroviral therapy. 2017. Available from: http://www.who.int/hiv/pub/guidelines/advanced-HIV-disease/en/.

WHO - World Health Organization. Guidelines for the diagnosis, prevention and management of cryptococcal disease in HIV-infected adults, adolescents and children. 2018. Available from: http:// www.who.int/hiv/pub/guidelines/cryptococcal-disease/en/.

WHO - World Health Organization. Rapid Advice: diagnosis, prevention and management of cryptococcal disease in HIV-infected adults, adolescents and children. Geneva: World Health Organization; 2011.

Zhai B, Wu C, Wang L, Sachs MS, Lin X. The antidepressant sertraline provides a promising therapeutic option for neurotropic cryptococcal infections. Antimicrob Agents Chemother. 2012; 56(7): 3758-66. 